\title{
Diferencia entre Ia alimentación complementaria clásica y el baby- led weaning en niños de 4 a 12 meses de edad
}

García-de la Cruz Paula Berenice*, González-Alcalá María Gabriela*, Jiménez-Vinatier Natalia*, Olivares-Pérez Carolina Elizabeth*, Ramírez-Reyes Tessy Paulina*, Sandoval-Solís Zaida Lizeth*, Pedroza-García Karina Alejandra*, Martín del Campo-Cervantes Judith ${ }^{* * *}$

\begin{tabular}{|c|c|}
\hline $\begin{array}{l}\text { Resumen } \\
\text { La introducción adecuada de los alimentos es importante en } \\
\text { la salud y crecimiento del lactante. Actualmente se pueden } \\
\text { seguir distintas técnicas como lo es el Baby-Led Weaning } \\
\text { (BLW), el cual, a diferencia de la técnica de alimentación } \\
\text { complementaria clásica, es autorregulado por el lactante. En } \\
\text { la Norma Oficial Mexicana NOM-043-SSA2-20 I 2, Servicios } \\
\text { básicos de Salud. Promoción y educación para la salud en } \\
\text { materia alimentaria, se establecen los criterios a seguir para } \\
\text { una correcta introducción de alimentos. Objetivo: comparar } \\
\text { el peso y la longitud en los lactantes que siguieron la alimen- } \\
\text { tación complementaria clásica y el BLW, así como los índi- } \\
\text { ces antropométricos. Material y métodos: estudio descriptivo } \\
\text { comparativo longitudinal realizado en 26 lactantes divididos } \\
\text { en dos grupos; el primero siguió la técnica de alimentación } \\
\text { complementaria clásica y, el segundo, la técnica de BLW; se } \\
\text { realizaron tres mediciones mensuales de peso y talla para } \\
\text { comparar y evaluar los índices antropométricos peso/longitud } \\
\text { (PIL), peso/edad (PIE) y longitudledad (L/E). Resultados: no se } \\
\text { encontraron diferencias significativas al comparar los índices } \\
\text { antropométricos de ambas técnicas de alimentación; por lo } \\
\text { que ambos métodos son recomendables. Se sugiere que se } \\
\text { realicen más estudios con una muestra más grande y conside- } \\
\text { rar otras variables como el desarrollo psicomotor. LUXMÉDICA } \\
\text { AÑo 15, NúmER0 45, SEPTIEMBRE-DICIEMBRE 2020, PP 73-80. }\end{array}$ & $\begin{array}{l}\text { Abstract } \\
\text { The proper introduction of food is important in the health } \\
\text { and growth of the infant. Currently, different techniques } \\
\text { can be followed, such as Baby-Led Weaning (BLW), which, } \\
\text { unlike the classic complementary feeding technique, is self- } \\
\text { regulated by the infant. The Official Mexican Norm NOM- } \\
\text { 043-SSA2-20 I2, Basic Health Services. Promotion and } \\
\text { education for health in food matters establishes the criteria } \\
\text { to be followed for a correct introduction of food. Objective: } \\
\text { To compare the weight and length in infants who followed } \\
\text { classical complementary feeding and BLW, as well as anthro- } \\
\text { pometric indices. Methods: This is a descriptive, comparative, } \\
\text { and longitudinal study carried out in } 26 \text { infants divided into } \\
\text { two groups; the first followed the classical complementary } \\
\text { feeding technique and the second the BLW technique. Three } \\
\text { monthly measurements of weight and height were made to } \\
\text { compare and evaluate the anthropometric indices weight/ } \\
\text { length (WIL), weight/age (WIA), and length/age (L/A). Re- } \\
\text { sults: No significant differences were found when comparing } \\
\text { the anthropometric indices of both feeding techniques; the- } \\
\text { refore, both methods are recommended. Conclusions: Further } \\
\text { studies are recommended with a larger sample, considering } \\
\text { other variables such as psychomotor development. LUXMÉDI- } \\
\text { CA AÑ 15, NúMER0 45, SEPTIEMBRE-DICIEMBRE 2020, PP 73-80. }\end{array}$ \\
\hline $\begin{array}{r}\text { Palabras clave: Alimentación complementaria clásica, } \\
\text { Baby-Led Weaning, lactante. }\end{array}$ & $\begin{array}{l}\text { Keywords: classical complementary feeding, baby-led } \\
\text { weaning, infant }\end{array}$ \\
\hline
\end{tabular}

* Estudiantes de la Licenciatura en Nutrición del Centro Ciencias de la Salud de la Universidad Autónoma de Aguascalientes, Aguascalientes, Orcid: https://orcid.org/0000-00024286-6182, https://orcid.org/0000-0002-3897-8886, https://orcid.org/0000-0002-3755-2699, https://orcid.org/0000-0002-1700-0790, https://orcid.org/0000-0001-7651-9325, https:// orcid.org/0000-0002-9128-6707 Correo electrónico: nataliajimvin@ @otmail.com

** Correo electronico: nataliajimvin@ hotmail.com México; Facultad de Ciencias Naturales de la Universidad Autónoma de Querétaro, Querétaro, México. https://orcid.org/0000-0001-6428-1743

*** Profesor investigador Titular B del Departamento de Nutrición y Cultura Física, Centro Ciencias de la Salud de la Universidad Autónoma de Aguascalientes, Aguascalientes, México. https://orcid.org/0000-0003-1855-6309

Fecha de recibido: 15 de octubre 2019 Fecha de aceptación: 26 de febrero 2020

Correspondencia: Dra. Judith Martín del Campo Cervantes. Departamento de Nutrición y Cultura Física Centro de Ciencias de la Salud. Universidad Autónoma de Aguascalientes. Av. Aguascalientes No.904, Edificio 107, Ciudad Universitaria, Aguascalientes, Ags. México CP 20131. Tel: +52 (449) 9107400 ext. 53355. Correo electrónico: jmartic@correo.uaa.mx 


\section{Introducción}

La alimentación complementaria es definida según la Norma Oficial Mexicana NOM-043-SSA2-2012 Servicios básicos de salud. Promoción y educación para la salud en materia alimentaria, como: "Un proceso que se inicia con la introducción gradual y paulatina de alimentos diferentes a la leche humana, para satisfacer las necesidades nutrimentales del niño o niña, se recomienda después de los 6 meses de edad".

Un punto clave en el inicio de la alimentación complementaria son las características con las que debe contar el lactante, las recomendaciones basadas en la Organización Mundial de la Salud (OMS) establecen que es primordial la maduración de las funciones gastrointestinales, renales y neurológicas. En las funciones digestivas, el lactante debe tener la capacidad de succión con reflejo de cierre de glotis, así como la desaparición del movimiento de extrusión, para que así permita la deglución de sólidos; estos movimientos desaparecen en el cuarto o quinto mes de vida. En cuanto a la función renal tiene que haber una filtración óptima, mientras que, para función neurológica, es necesario que el lactante pueda sentarse por sí solo, barra con su labio superior el contenido de una cuchara y pueda deglutir el alimento sólido; estas dos funciones se pueden lograr a partir de los seis meses de vida., ${ }^{2,3}$

El método denominado como alimentación complementaria clásica es el comúnmente utilizado, en el cual la alimentación es guiada por el responsable del lactante por medio de una cuchara; se inicia con papillas y las texturas de los alimentos se modifican gradualmente. $^{4}$

Las ventajas del método de la alimentación complementaria clásica es que permite el desarrollo del sentido del gusto por nuevos alimentos, la adquisición de hábitos alimentarios para que, en un futuro, se cree una óptima aceptación de una dieta variada y el desarrollo de estructuras bucales. Sus desventajas son que, si se inicia de manera precoz puede aumentar riesgo a enfermedades respiratorias, produce daño renal por sobrecarga de nutrientes, aumenta riesgo de desarrollar hipertensión arterial, obesidad, diabetes mellitus y enfermedades cardiovasculares. Por otra parte, si su inicio es tardío puede provocar deficiencias de hierro, vitamina $A$, zinc, crecimiento lento del lactante, trastornos de rechazo de alimentos y alergias. ${ }^{5-7}$

Sin embargo, en los últimos años ha crecido en popularidad un enfoque alternativo conocido como Baby-Led Weanind (BLW), el cual indica que los lactantes pueden autoalimentarse. El énfasis está en permitir que el lactante elija qué y cuánto comer $y$, así mismo, comience a integrarse a la alimentación familiar. ${ }^{8}$ El BLW fue creado por Rapley y Murkett; está basado en la observación del lactante y su capacidad de alimentarse por sí mismo, así como elegir el ritmo de introducción de alimentos sólidos, en lugar de ser alimentados por el responsable haciendo uso de la cuchara. Su 


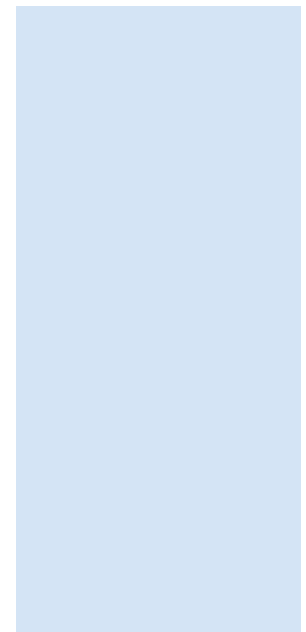

objetivo es hacer el proceso de alimentación más placentero mediante la incorporación precoz del lactante a la dinámica familiar de la alimentación. 9,10

Algunas ventajas de esta técnica son que puede contribuir a un mejor desarrollo de la saciedad, así como disminuir la aparición de obesidad por la autorregulación y cambiar las preferencias de alimentación hacia pautas más saludables. También existen ciertas desventajas como son la probabilidad de una ingesta de energía insuficiente, riesgo de atragantamiento y probable alergia alimentaria. ${ }^{10}$

Existen pocos estudios que comparen la alimentación complementaria clásica con el BLW, por lo que en la presente investigación se comparó el peso y la longitud en la alimentación complementaria clásica y el BLW, aplicada en niños de 4 a 12 meses de edad.

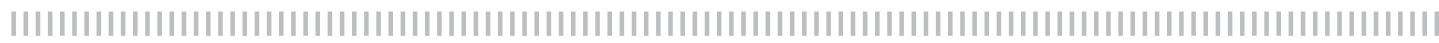

\section{Material y métodos}

Se realizó un estudio descriptivo, comparativo y longitudinal durante enero a junio de 2018, en un muestreo no probabilístico por conveniencia de 26 lactantes de Aguascalientes, Ags. México, con las siguientes características: de 4 a 12 meses de edad, que se mantuvieran sentados por sí solos, que no presentaran reflejo de extrusión, que presentaran control de sostén de la cabeza y que mostraran interés por los alimentos.

Se comenzó a buscar e informar a madres con hijos que cumplieran con las características antes expuestas y también se asistió a una Estancia de Bienestar y Desarrollo Infantil. Se les explicó a los tutores del lactante el objetivo de la investigación, así como las dos formas de alimentación complementaria y, si los tutores aceptaban que su hijo ingresara a la investigación, se les pedía que firmaran un consentimiento informado.

Se dividió la muestra en dos grupos, el primero siguió la técnica de alimentación complementaria clásica de la NOM-043, Servicios básicos de Salud. Promoción y educación para la salud en materia alimentaria; ${ }^{1}$ y en el segundo grupo se aplicó la técnica de Baby-Led Weaning (BLW), con base en las indicaciones que dan la OMS y NOM-043. ${ }^{1,2}$
A los padres de BLW se les pidió que asistieran a un taller en donde se les explicó la técnica de maniobra que se llevaría durante los tres meses de la investigación; además, se dio una explicación de forma teórica en la elaboración y preparación de los alimentos, así como del tamaño de la porción del alimento que se le ofrecería al lactante; aunado a esto se les explicó cómo evitar el riesgo de atragantamiento y qué hacer en caso de que ocurriera. Mientras que, a los padres de alimentación complementaria clásica se les impartió un taller de la forma para introducir los alimentos de acuerdo a lo descrito en la NOM-043, Servicios básicos de Salud. Promoción y educación para la salud en materia alimentaria.

Se mantuvo comunicación periódica con ambos grupos con la finalidad de resolver dudas o problemáticas que se pudieran presentar durante el proceso de introducción de alimentos.

Mediciones antropométricas: se realizaron tres mediciones mensuales con base en las técnicas de antropometría del Instituto Nacional de Alimentación y Nutrición. ${ }^{11}$ El peso de los lactantes fue tomado con básculas estandarizadas como la báscula de impedancia bioeléctrica marca Tanita modelo UM-061, la báscula Inner Scan Body Composition marca Tanita modelo BC 553 
- la báscula marca Shekel. Para la medición de longitud de los lactantes se utilizó un infantómetro marca SECA, modelo 210. Los índices evaluados fueron peso para la longitud $(P / L)$, peso para la edad $(P / E)$ y longitud para la edad (L/E) de acuerdo con los patrones de crecimiento infantil de la
OMS. ${ }^{12-17}$ Análisis estadístico: se utilizó el programa SPSS versión 20 para el análisis de los datos, se compararon los grupos mediante la prueba $t$ de student para muestras independientes considerando un nivel de significancia con valor de $p<0.05$.

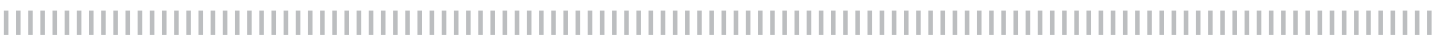

\section{Resultados}

Se observó que el promedio del peso inicial fue de $8.0 \mathrm{~kg}$ y la longitud fue de 69.48 $\mathrm{cm}$; al término de estudio la media poblacional de peso fue de $8.85 \mathrm{~kg}$ y de longitud fue de $71.94 \mathrm{~cm}$ (Tabla 1).

\section{Tabla I}

\section{Características antropométricas poblacionales y por grupo de estudio}

\begin{tabular}{|c|c|c|c|}
\hline $\begin{array}{l}\text { Evaluaciones } \\
\text { antropométricas }\end{array}$ & $\begin{array}{l}\text { Población en general } \\
\text { media } \pm \text { DE }\end{array}$ & $\begin{array}{c}\text { Alimentación } \\
\text { complementaria clásica } \\
\text { media } \pm \text { DE }\end{array}$ & $\begin{array}{l}\text { Baby-Led Weaning } \\
\text { media } \pm \text { DE }\end{array}$ \\
\hline \multicolumn{4}{|c|}{ Primera evaluación } \\
\hline Peso (kg) & $8.0+1.12$ & $7.95 \pm 0.70$ & $8.05 \pm 1.45$ \\
\hline Longitud $(\mathrm{cm})$ & $69.48+3.32$ & $69.07 \pm 2.38$ & $69.88 \pm 4.11$ \\
\hline Peso/Edad & $37.57+26.30$ & $36.46 \pm 25.77$ & $38.69 \pm 27.83$ \\
\hline Longitud / Edad & $40.38+21.22$ & $35.23 \pm 17.55$ & $45.54 \pm 23.93$ \\
\hline Peso/ Longitud & $38.69+29.43$ & $41.62 \pm 29.83$ & $35.77 \pm 29.94$ \\
\hline \multicolumn{4}{|c|}{ Segunda evaluación } \\
\hline Peso (kg) & $8.44+1.44$ & $8.10 \pm 0.76$ & $8.79 \pm 1.87$ \\
\hline Longitud (cm) & $70.63+3.51$ & $70.19 \pm 2.66$ & $71.07 \pm 4.26$ \\
\hline Peso/Edad & $41.80+29.49$ & $33.69 \pm 21.46$ & $49.92 \pm 34.59$ \\
\hline Longitud /Edad & $39.84+26.10$ & $32.54 \pm 22.81$ & $47.15 \pm 27.99$ \\
\hline Peso/ Longitud & $43.26+28.77$ & $33.69 \pm 21.75$ & $52.85 \pm 32.64$ \\
\hline \multicolumn{4}{|l|}{ Tercera evaluación } \\
\hline Peso (kg) & $8.85+1.19$ & $8.94 \pm 0.91$ & $8.76 \pm 1.45$ \\
\hline Longitud (cm) & $71.94+3.02$ & $71.50 \pm 1.97$ & $72.38 \pm 3.83$ \\
\hline Peso/Edad & $48.26+29.42$ & $49.23 \pm 25.96$ & $47.31 \pm 33.57$ \\
\hline Longitud / Edad & $36.34+25.57$ & $30.77 \pm 19.56$ & $41.92 \pm 30.20$ \\
\hline Peso/ Longitud & $47.11+32.61$ & $51.69 \pm 33.37$ & $42.54 \pm 32.51$ \\
\hline
\end{tabular}

DE: Desviación estándar.

$n=26$ lactantes (13 lactantes en alimentación complementaria clásica y 13 en Baby-Led Weaning) 
Posteriormente se comparó el peso, la longitud y los indicadores: peso para la longitud, longitud para la edad y peso para la edad final de los lactantes que siguieron la técnica de alimentación com- plementaria clásica contra la técnica de baby-led weaning, no encontrando diferencias significativas entre los grupos, véase los resultados en la Tabla 2.

\section{Tabla 2}

\section{Comparación del peso longitud e índices antropométricos finales de lactantes alimentados con alimentación complementaria clásica y Baby-Led Weaning}

\begin{tabular}{|c|c|c|c|}
\hline $\begin{array}{c}\text { Evaluaciones } \\
\text { antropométricas }\end{array}$ & $\begin{array}{c}\text { Alimentación } \\
\text { complementaria clásica } \\
\text { media } \pm \text { DE }\end{array}$ & $\begin{array}{c}\text { Baby-Led Weaning } \\
\text { media } \pm \text { DE }\end{array}$ & $\boldsymbol{p}$ \\
\hline Talla $(\mathrm{cm})$ & $71.5 \pm 1.98$ & $72.38 \pm 3.84$ & 0.469 \\
\hline Peso (kg) & $8.95 \pm 0.91$ & $8.76 \pm 1.45$ & 0.703 \\
\hline Peso/Talla & $51.69 \pm 33.37$ & $42.54 \pm 32.51$ & 0.486 \\
\hline Talla/Edad & $30.77 \pm 19.56$ & $41.92 \pm 30.20$ & 0.277 \\
\hline Peso/Edad & $49.23 \pm 25.96$ & $47.32 \pm 33.57$ & 0.872 \\
\hline
\end{tabular}

Valores de p utilizando la prueba t de student para muestra independiente, $p<0.05$. $n=13$ lactantes en alimentación complementaria clásica y 13 en Baby-Led Weaning.

Al realizar una revisión de la media del peso, se encontró un mayor aumento de peso en la segunda evaluación para el grupo de BLW, el cual se mantuvo para el tercer mes; mientras que el grupo de alimentación complementaria clásica lo presentó en la última medición (figura 1). El aumento para el grupo de BLW fue de $0.71 \mathrm{~kg}$ en el periodo de evaluación, mientras que en el grupo de alimentación complementaria clásica el aumento fue de $0.99 \mathrm{~kg}$ en el mismo periodo.

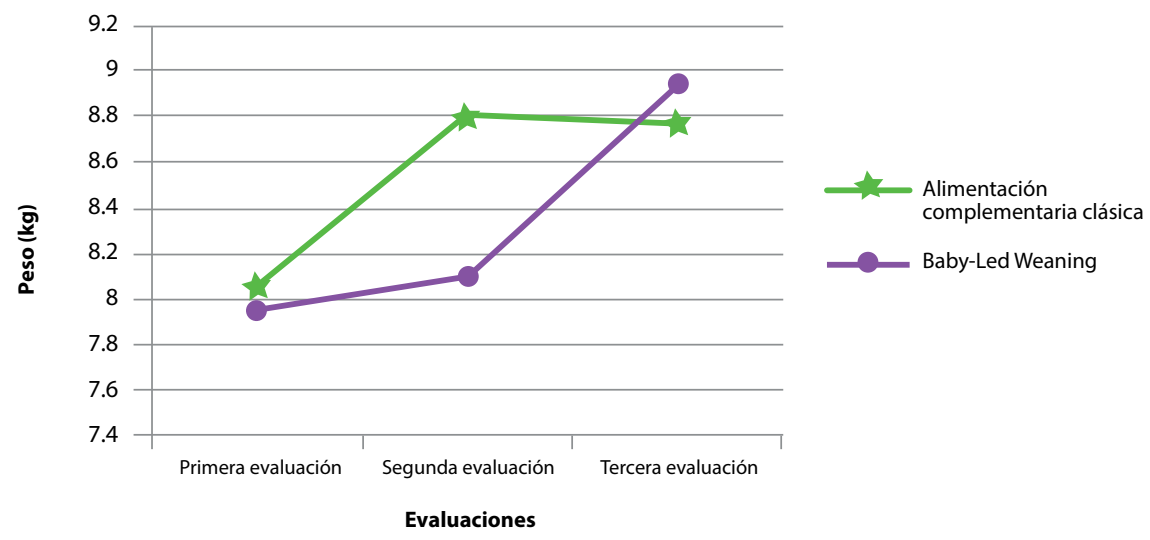

Figura 1. Comparación del peso longitud e índices antropométricos de lactantes alimentados con alimentación complementaria clásica y Baby-Led Weaning $n=13$ lactantes en alimentación complementaria clásica y 13 en Baby-Led Weaning 


\section{Discusión}

De acuerdo con el estudio realizado no se encontró diferencia significativa en el indicador de peso para la longitud, lo cual es contrario a los beneficios del método BLW mencionado por Vega que menciona que los lactantes alimentados siguiendo el método de BLW desarrollan gustos por alimentos más sanos y presentan una menor prevalencia de obesidad, lo cual se atribuye a la capacidad de autorregularse permitiendo el correcto funcionamiento de los mecanismos del hambre y la saciedad. ${ }^{18}$ Townsend y Pitchford realizaron un estudio en Reino Unido, en donde encontraron que el BLW se asocia a un menor índice de masa corporal en los lactantes y se concluye que estas prácticas pueden ser una forma de combatir la obesidad infantil. ${ }^{19}$ Otro beneficio al que se hace referencia con el método de BLW es el desarrollo de la musculatura orofacial; favoreciendo una correcta masticación y posteriormente una mayor capacidad para hablar. Además de que promueve el desarrollo de la motricidad fina y la coordinación visomotora. ${ }^{18}$

Por otro lado, Rowan y Harris indicaron que los lactantes a los que se les introducen los alimentos con el método de BLW comparten la hora de comida con la familia, lo cual puede favorecer puntos negativos asociados a los hábitos alimentarios de la familia, como es que los alimentos pueden ser altos en sodio, azúcar y grasas saturadas y bajas en energía y folatos. ${ }^{7}$

Según la Organización Mundial de la Salud, es fundamental que la introducción de la alimentación complementaria se haga con alimentos "apropiados, suficientes y seguros" y que se cubran los requerimientos energéticos del niño tanto de forma cuantitativa como cualitativa. El periodo transicional debe comenzar no antes de los cuatro meses ni después de los seis, tal y como afirma la Sociedad Europea de Gastroenterología y Nutrición. En la realización de este estudio, se hicieron observaciones a algunos de los padres de lactantes de la muestra que habían comenzado la alimentación complementaria antes de esta edad o después, 20,21 explicándoles las posibles repercusiones que dicha acción podía haber generado en la salud de sus hijos.

Por otro lado, el Departamento de Salud del Reino Unido recomienda comer con los dedos desde el comienzo del destete para que, de esta manera, se incorpore al lactante a la alimentación familiar; al realizar un estudio de cohorte retrospectivo, se mostró que hasta el $96 \%$ de los lactantes entre siete a ocho meses de edad buscan utilizar sus dedos para consumir los alimentos; sin embargo, los autores señalan que esto puede ser una subestimación ya que dependía de qué alimento se ofreciera a los lactantes. ${ }^{8}$

En el presente estudio se mostró que no hubo cambios en los parámetros e indicadores antropométricos entre un método de alimentación y otro; se cree que esto se debió a que ambos grupos siguieron el mismo esquema de introducción de alimentos, mismo que estuvo basado en la NOM-043; lo único que cambió fue la textura y el papel que jugó el lactante en cada método, por lo que la cantidad y la calidad del alimento fue la misma, dando así el mismo aporte nutrimental.

Dentro del estudio se tuvieron ciertas limitaciones, las cuales pudieron afectar los resultados; como lo fue un tamaño de muestra pequeño, además de que la edad de los niños fue muy variada, siendo que algunos de ellos ya habían iniciado su alimentación complementaria; también se observó que hubo poca participación y compromiso por parte de los responsables de los lactantes; por otro lado, se cree que el tiempo de observación fue un periodo muy corto, por lo que se sugiere extender mínimo hasta el año de edad para la obtención de mejores resultados.

Otra limitante fue el no poder llevar un control individual de la dieta como para generar un análisis global de ésta; sin embargo, se recomienda llevar un control in- 
dividualizado de la dieta por lactante para que el aporte de nutrimentos sean los mismos en todos y así obtener un resultado más confiable.

Aun con los resultados obtenidos y de acuerdo con la bibliografía consultada, se considera que el BLW es una mejor técnica de alimentación complementaria, ya que implica que los lactantes se autorregulen de acuerdo con el mecanismo del hambre y la saciedad; lo cual puede influir de manera significativa en los problemas de obesidad que se pudieran presentar a lo largo de su vida. Aunque en los resultados se vio que no hubo una diferencia significativa entre un método y otro, se cree que, si se mejoran las limitaciones anteriormente mencionadas, se podría llegar a comprobar que el BLW tiene un mejor efecto que la alimentación complementaria clásica, considerando que se realicen más estudios.

\section{Conclusiones}

Tras la comparación de la ablactación clásica y el Baby-Led Weaning se encontró que no hubo diferencia significativa en los índices antropométricos, por lo que ambos métodos son igual de recomendables. Se sugiere que se realicen más estudios con una muestra más grande y se consideren otras variables como el desarrollo psicomotor.

\section{Agradecimientos}

Se agradece el apoyo a las instituciones de Estancia de Bienestar y Desarrollo Infantil del Instituto de Seguridad y Servicios Sociales para los Trabajadores del Estado (ISSSTE) No. 21 y No. 102, y al Departamento de Nutrición y Cultura Física de la Universidad Autónoma de Aguascalientes por el préstamo de las instalaciones y del equipo antropométrico, así como a las madres de familia que participaron en la investigación.

\section{Declaración de autores}

Los autores declaran que no existe conflicto de interés.

\section{Bibliografía}

1. Secretaria de Salud [Web]. Norma Oficial Mexicana NOM-043-SSA2-2012, Servicios básicos de salud. Promoción y educación para la salud en materia alimentaria. Criterios para brindar orientación; 24 diciembre 2001 [actualizado 2012; citado Mayo 2018]. Disponible en: http://www.salud.gob.mx/ unidades/cdi/nom/compi/043ssa205.pdf

2. Organización Mundial de la Salud (OMS) [Web]. Alimentación complementaria; [citado mayo 2018]. Disponible en: http://www.who.int/nutrition/topics/complementary_feeding/es/

3. Daza W, Dadán S. Alimentación complementaria en el primer año de vida. Precop SCP CCAP. 2017; 8(4): 18-27.
4. Rojas C, Guerrero R. Nutrición clínica y gastroenterología pediátrica. Bogotá (Colombia): Médica Panamericana; 1999

5. Organización Mundial de la Salud. La alimentación del lactante y del niño pequeño. Washington: Organización Panamericana de la Salud; 2010.

6. Realpe A, Ortiz H. Alimentación complementaria en el primer año de vida. Rev Gastro hnup. 2016; 18(1):43-47.

7. Rowan $\mathrm{H}$, Harris $\mathrm{C}$. Baby-led weaning and the family diet. A pilot study. Elsevier. 2012; 58 (3): $1046-$ 1049.

8. Brown A, Wyn S \& Rowan H. Baby-Led Weaning: The Evidence to Date. Curr Nutr Rep. 2017; (6):148-156 
9. Izquierdo $E$, Segoviano $M$, Verges $C$. Complementary feeding: what, when and how. Rev Pediatr Aten Primaria. 2016; 18(69): 31-35.

10. Colli G. El riego de atragantamiento y asfixia al hacer el Baby-Led Weaning (BLW). España; 9 Julio 2017 [citado mayo 2018]. Disponible en: http://www. criarconsentidocomun.com/el-riesgo-de-atragantamiento-y-asfixia-al-hacer-el-baby-led-weaning-blw/

11. Instituto Nacional de Salud Centro Nacional de Alimentación y Nutrición. Medidas antropométricas, riesgo y estandarización. Lima; 1998 [citado Mayo 2018]. Disponible en: http://www.bvs.ins.gob.pe/ insprint/cenan/modulo_medidas_antropometricas registro estandarizacion.pdf

12. Organización Mundial de la Salud (OMS) [Web]. Longitud para la edad, niñas. 2008 [citado junio 2018]. Disponible en: http://www.who.int/childgrowth/standards/cht_Ifa_ninas_p_0_2.pdf?ua =1

13. Organización Mundial de la Salud (OMS) [Web]. Longitud para la edad, niños. 2008 [citado junio 2018]. Disponible en: http://www.who.int/childgrowth/standards/cht Ifa ninos p 0 2.pdf?ua $=1$

14. Organización Mundial de la Salud (OMS) [Web]. Peso para la edad, niñas. 2008 [citado junio 2018]. Disponible en: http://www.who.int/childgrowth/ standards/cht wfa ninas p 0 2.pdf?ua $=1$

15. Organización Mundial de Ta Salud (OMS) [Web].
Peso para la edad, niños. 2008 [citado junio 2018]. Disponible en: http://www.who.int/childgrowth/ standards/cht wfa ninos p 0 2.pdf?ua $=1$

16. Organización Mundial de la Salud (OMS) [Web]. Peso para la longitud, niñas. 2008 [citado junio 2018]. Disponible en: http://www.who.int/childgrowth/standards/cht_wfl_ninas_p_0_2.pdf?ua =1

17. Organización Mundial de la Salud (OMS) [Web]. Peso para la longitud, niños. 2008 [citado junio 2018]. Disponible en: http://www.who.int/childgrowth/standards/cht_wfl_ninos_p_0_2.pdf?ua $=1$

18. Vega Velasco Manrique $\bar{M}$. Alimentación complementaria guiada por el bebé: respetando sus ritmos y apoyando su aprendizaje. España. 2014; 8 (2): 6472.

19. Townsend E, Pitchford NJ. Baby knows best? The impact of weaning style on food preferences and body mass index in early childhood in a case- controlled sample. BMJ Open. 2012; 2.

20. Organización Mundial de la Salud. Lactancia materna exclusiva [Internet]. OMS; 2017. [citada 18 feb 2017]. Disponible en: http://www.who.int/nutrition/topics/exclusive_breastfeeding/es/

21. Almarza A.L. Martínez B. M. Alimentación del lactante sano. Protocolos diagnósticos- terapéuticos de Gastroenterología, Hepatología y Nutrición Pediátrica. SEGHP-AEP. 2010; 287-295. 